\title{
Assessment of local approach methods for predicting end- of-life toughness of RPV steels
}

DOI:

10.1115/PVP2011-57546

Link to publication record in Manchester Research Explorer

\section{Citation for published version (APA):}

Jivkov, A. P., Lidbury, D. P. G., \& James, P. (2011). Assessment of local approach methods for predicting end-oflife toughness of RPV steels. In American Society of Mechanical Engineers, Pressure Vessels and Piping Division (Publication) PVPIASME Pressure Vessels Piping Div. Publ. PVP (Vol. 6, pp. 293-302). American Society of Mechanical Engineers. https://doi.org/10.1115/PVP2011-57546

\section{Published in:}

American Society of Mechanical Engineers, Pressure Vessels and Piping Division (Publication) PVP|ASME Pressure Vessels Piping Div. Publ. PVP

\section{Citing this paper}

Please note that where the full-text provided on Manchester Research Explorer is the Author Accepted Manuscript or Proof version this may differ from the final Published version. If citing, it is advised that you check and use the publisher's definitive version.

\section{General rights}

Copyright and moral rights for the publications made accessible in the Research Explorer are retained by the authors and/or other copyright owners and it is a condition of accessing publications that users recognise and abide by the legal requirements associated with these rights.

\section{Takedown policy}

If you believe that this document breaches copyright please refer to the University of Manchester's Takedown Procedures [http://man.ac.uk/04Y6Bo] or contact uml.scholarlycommunications@manchester.ac.uk providing relevant details, so we can investigate your claim.

\section{OPEN ACCESS}




\section{ASSESSMENT OF LOCAL APPROACH METHODS FOR PREDICTING END-OF-LIFE TOUGHNESS OF RPV STEELS}

\author{
Andrey P Jivkov \\ The University of Manchester \\ Manchester, UK
}

\author{
David P G Lidbury \\ Serco Technical Consulting \\ Services \\ Risley, Warrington, UK
}

\author{
Peter James \\ Serco Technical Consulting \\ Services \\ Risley, Warrington, UK
}

\begin{abstract}
Local approach methods are becoming increasingly popular as practical tools for cleavage fracture toughness prediction. Their application involves two distinct elements: calculation of 'individual' probabilities of failure, dictated by the local mechanical fields; and summation of these failure probabilities to predict the probability of component failure. In this work, we demonstrate that development of the local approach methods to date has been essentially focused on improving the criterion for predicting local failure as a function of the local mechanical fields. Yet, the existing methods fail to predict with sufficient accuracy the effects of irradiation and defect geometry on fracture toughness when the calculations are based on a common set of model parameters. A possible reason for this, common to all methods, is found in the calculation of the cumulative failure probability, which is based on the weakestlink argument. We discuss the implications of the weakest-link assumption, identify those situations where it needs to be reconsidered, and propose future work that will increase our understanding for improving the calculation of global failure probability.
\end{abstract}

\section{INTRODUCTION}

The ability to predict changes in cleavage fracture toughness behaviour of ferritic RPV steels accounting for the effects of irradiation and defect geometry is important to safety assessment and life extension decisions in nuclear plant. Local approaches to cleavage fracture offer a promising methodology to help achieve this. In principle, these are based on current understanding of the micro-mechanisms involved in the cleavage failure phenomenon, such as the nucleation of microcracks at second-phase brittle particles, the propagation of such micro-cracks within grains and the propagation of a critical micro-crack leading to component failure. The scope of this work does not include local approaches that involve changes in the material constitutive behaviour as a result of local damage, i.e. micro-cracking and/or void growth and coalescence prior to cleavage. The local approaches considered are those based on the calculation of the component probability of failure as a function of the mechanical fields in the material undergoing elastic-plastic deformations consistent with the macroscopically measured tensile behaviour. This allows for the local approaches considered to be implemented in a post-processing routine.

The existing local approach methods share a common philosophy based on two distinct components. In the first place, they assume that the local mechanical fields provide a local or 'individual' probability of failure when linked to the size distribution of the second-phase particles capable of microcrack nucleation. The individual probability of failure at location $i$ can be written in the following general form

$$
p_{f, i}=\int_{r_{c, i}}^{\infty} p_{c, i} f(r) d r
$$

where $f(r)$ is the probability density of particle sizes associated with the nucleation of micro-cracks, $p_{c, i}$ is the probability of micro-crack nucleation at location $i$, and $r_{c, i}$ is a critical microcrack size at location $i$. Note that $p_{c, i} f(r)$ is the probability density of nucleated micro-crack sizes at location $i$. All existing methods can be recast in the form (1) albeit varying in their definitions of $p_{c}$ and $r_{c}$, which will be discussed shortly.

In the second place, the existing methods assume that the individual failure events in the component are independent. This allows the weakest-link argument to be invoked for calculating the global probability of failure. According to this, the global failure probability is one minus the global probability of survival, which is the product of all individual survival probabilities, i.e. 


$$
P_{f}(V)=1-\prod_{i=1}^{N}\left(1-p_{f, i}\right),
$$

where $N$ is the number of possible weakest-links, i.e. active micro-cracks, in the relevant volume $V$. In practice, the local approaches are applied to finite element solutions of cracked components, where the mechanical fields have constant values within the volume of one integration point. According to (2) the probability of failure of one such volume $V_{i}$ is given by

$$
P_{f}\left(V_{i}\right)=1-\left(1-p_{f, i}\right)^{N_{i}}=1-\left(1-p_{f, i}\right)^{\rho_{i} V_{i}},
$$

where $N_{i}=\rho_{i} V_{i}$ is the number of micro-cracks in $V_{i}$, with $\rho_{i}$ being the density of the micro-cracks, which equals the density of particles, $\rho$, times the probability of particle failure, $p_{c, i}$. The component probability of failure is then calculated by repeated application of (2) to arrive at

$$
P_{f}(V)=1-\prod_{i=1}^{I P}\left(1-p_{f, i}\right)^{\rho_{i} V_{i}}
$$

where the product is taken over all relevant integration points.

The aim of this work is to assess the improvements of the local approach models proposed over the years. We discuss the expressions for the individual probability of failure and the weakest-link approach to obtain the global probability of failure. Under certain assumptions, the global probability of failure can be written as a Weibull distribution function of a global stress parameter, called the Weibull stress. The majority of the existing local approach methods belong to this class. With several Weibull stress-based models we present the predictions for the fracture toughness changes due to irradiation and loss of constraint, separately and simultaneously, as well as the changes in the model parameters with temperature required for these predictions to conform to known experimental data. We demonstrate that predictions for fracture toughness changes due to irradiation and constraint effects cannot be made with a common set of models' parameters and discuss possible reasons for this.

We present also a new micro-mechanically informed model for the local probability of failure, where the weakest-link argument is used directly to calculate the global probability of failure. In this case, a Weibull stress is not defined. This model provides better predictions for experimentally observed spatial distribution of cleavage initiation sites compared to the Weibullstress models. The model is also used to predict the cleavage fracture toughness temperature dependence. The prediction is somewhat better than that of correspondingly constrained Weibull-stress models. The results suggest that the improvements introduced in the calculation of individual failure probabilities are not sufficient for predicting global probability of failure, the reason for this originating in current implementations of the weakest-link argument. The assumption that the individual failure events are independent becomes increasingly impractical with increasing plasticity, either with load increases at a fixed temperature or with a change in yield stress across temperatures. We conclude that future work should focus on understanding the interactions between microcracks to develop a more physically realistic basis for performing cumulative probability of failure calculations.

\section{CRACK ANALYSIS}

A boundary layer model is used to analyse the stress and strain fields ahead of a macro-crack. The geometry of the model consists of a semi-circular disk centred at the crack tip. The crack is modelled as a long, slender notch with a circular root. The ratio between the disk radius, $R$, and the root radius, $r$, is $10^{5}$. This arrangement facilitates large strain analysis, which is an essential prerequisite for the realistic application of the local approach models. Analyses were performed with ABAQUS [1] using plane strain isoparametric quadrilateral elements with linear interpolation.

The material studied is an RPV $22 \mathrm{NiMoCr} 37$ ferritic steel, known as Euro Material A, for which we have the mechanical and fracture toughness properties at start of life and after irradiation at a temperature of $285^{\circ} \mathrm{C}$ to a fluence of $4.3 \times 10^{19}$ $\mathrm{n} / \mathrm{cm}^{2}$ from the Euratom FP6 project PERFECT [2]. The temperature dependence of Young's modulus $E$, proportionality stress $\sigma_{0}$, and ultimate tensile strength $\sigma_{u}$, at start of life are

$$
\begin{aligned}
& E=-90 T+206000, \\
& \sigma_{0}=421.2+63.9 \exp (-T / 91), \\
& \sigma_{u}=564.1+70.2 \exp (-T / 108),
\end{aligned}
$$

where $T$ is in ${ }^{\circ} \mathrm{C}$ and $E, \sigma_{0}$, and $\sigma_{u}$ are in MPa. In the irradiated condition (5) is considered not to change, while $\sigma_{0}$ and $\sigma_{u}$ are given by

$$
\begin{aligned}
& \sigma_{0}=490.4+62.1 \exp (-T / 95.2), \\
& \sigma_{u}=524.2+155.1 \exp (-T / 188.2) .
\end{aligned}
$$

Poisson's ratio is $v=0.3$, assumed independent of temperature and irradiation. The material behaviour is represented with a power-law, where the strain is elastic for $\sigma<\sigma_{0}$ and depends on $\sigma^{n}$ for $\sigma>\sigma_{0}$. The hardening exponent $n$ is determined from (6)-(7) or (8)-(9) for a given $T$ using Considiere's rule.

The fracture toughness properties of Material A given in [2] were determined according to the ASTM standard [3] using SEN(B) specimens in three-point bending. This standard is based on the Master Curve formalism, which defines the temperature dependence of a reference toughness, $K_{0}$, relative to a reference temperature, $T_{0}$, at which $K_{0}=108 \mathrm{MPa} \sqrt{\mathrm{m}}$, for high-constraint cracked geometries with reference crack front length $B_{0}=25.4 \mathrm{~mm}$

$$
K_{0}=31+77 \exp \left[0.019\left(T-T_{0}\right)\right] .
$$


The scatter in measured cleavage fracture toughness values is then described as a function of the probability of failure, $p$, and the actual crack front length, $B$, with

$$
K_{p}=K_{\min }+\left(K_{0}-K_{\min }\right)\left(\frac{B_{0}}{B}\right)^{1 / 4}\left(\ln \frac{1}{1-p}\right)^{1 / 4}
$$

where $K_{\min }$ is a temperature independent threshold toughness $\left(K_{\min }=20 \mathrm{MPa} \sqrt{\mathrm{m}}\right.$ according [3]). According to the Master Curve formalism, (10) and (11) define fully the cleavage fracture toughness behaviour of a material with known $T_{0}$.

The reference temperature for unirradiated deep-notch $\left(a_{0} / W=0.5\right)$ specimens reported in [2] is $T_{0}=-104^{\circ} \mathrm{C}$. The reference temperature for unirradiated shallow-notch $\left(a_{0} / W=0.1\right) \quad$ specimens is $T_{0}=-128^{\circ} \mathrm{C}$. The reference temperature shift due to low constraint is thus $\Delta T_{0}=-24^{\circ} \mathrm{C}$. The reference temperature for irradiated deep-notch $\left(a_{0} / W=0.5\right)$ specimens is $T_{0}=-78^{\circ} \mathrm{C}$. The reference temperature shift due to irradiation is thus $\Delta T_{0}=26^{\circ} \mathrm{C}$. In the present work, we assume that (10) and (11) provide a relevant representation of experimental data and assess the local approach models discussed against its predictions.

The boundary layer model is loaded by prescribed displacements along the circular boundary, given by the William's expansions [4]. For the models representing deep notch specimens, the expansion contains only the singular term which depends on the Mode I stress intensity factor, $K_{I}$. For the models representing shallow notch specimens, the expansion contains the first non-singular term which depends on the elastic $T$-stress. In these cases, the $T$-stress is calculated as a function of $K_{I}$, according to available solutions for three-point bend specimens with $a_{0} / W=0.1$ [5]. The models are analysed at a number of temperatures in the range $T_{0}-75^{\circ} \mathrm{C}<T<T_{0}+75^{\circ} \mathrm{C}$. At each of these, the load is increased to an applied stress intensity corresponding to the $99 \%$ fracture toughness found from (11).

\section{WEIBULL-STRESS BASED METHODS}

The first local approach method for cleavage fracture modelling was proposed by the Beremin group [6]. This method recognised that plastic strains in the matrix produce overload in the brittle second-phase particles and are a necessary prerequisite for their failure. It was therefore assumed that $p_{c}=1$ in the presence of plastic strains and $p_{c}=0$ otherwise. This means that particles are assumed to fail within the plastic zone ahead of a macro-crack with equal probability and to remain intact outside the plastic zone. The integrand in (1) is the probability density of the second-phase particles sizes and this is equal to the probability density of the nucleated micro-crack sizes. We will refer to this model as $\langle\mathrm{M} 0\rangle$.

Later developments of the Beremin model suggested that the probability of particle failure is proportional to the plastic strains level, typically via the equivalent plastic strain, see e.g. $[7,8]$, i.e.

$$
p_{c}=C \varepsilon_{p}^{e q}
$$

where $C$ is a positive constant. This suggestion is supported by experimental observations that larger plastic strains increase the probability of particle failure, other conditions being equal. The linear relation between equivalent plastic strain and the probability of particle failure is not fully justified but is considered as an acceptable first approximation. We will refer to this model as $\langle\mathrm{M} 1\rangle$.

In the latest developments of the Beremin model it was recognised that the stress triaxiality at a particle location might also play an important role in particle failure probability. A recent model incorporating the roles of plastic strains and stress triaxiality in particle failure probability is reported in [9]:

$$
p_{c}=C_{1} \varepsilon_{p}^{e q} \exp \left(-C_{2} \frac{\sigma_{h}}{\sigma_{e q}}\right)
$$

where $C_{1}$ and $C_{2}$ are positive constants, and $\sigma_{h}$ and $\sigma_{e q}$ are the hydrostatic and the equivalent stresses, respectively. We will refer to this model as $\langle\mathrm{M} 2\rangle$ and will assume $C_{2}=1.5$, as suggested in [9].

It should be noted that any of the above models is amenable to direct formulation, i.e. in terms of the current stress and strain fields, and to a corresponding incremental formulation, similar to the Bordet model [10]. The common feature of these models is that the probability of particle failure is independent of the particle size. This means that $p_{c}$ becomes a factor in the integral in (1), which in such cases is determined only from the probability density of particle sizes. In other words, the local $p_{c}$ in the more advanced models $\langle\mathrm{M} 1\rangle$ and $<$ M2> multiplies the individual probability of failure that is determined via the original Beremin model. When the probability density of second-phase particle sizes is assumed to obey a power-law, such as

$$
f(r)=\frac{\alpha-1}{r_{0}}\left(\frac{r}{r_{0}}\right)^{-\alpha}
$$

then the local probability of failure (1) has a closed-form expression in terms of critical micro-crack sizes

$$
p_{f, i}=p_{c, i}\left(\frac{r_{c, i}}{r_{0}}\right)^{1-\alpha} .
$$

In (14) and (15), $\alpha$ is the shape of the particle size distribution and $r_{0}$ is the minimum (cut-off) particle size. The power-law distribution is the assumption in the Weibull-stress based models because (15), combined with the weakest-link statistics (4), provides a global probability of failure expressed as a Weibull distribution. To accommodate this, it is further 
assumed that the individual failure probabilities are small, so that $\left(1-p_{f, i}\right) \approx \exp \left(-p_{f, i}\right)$. This, taken with (4) and (15), provides

$$
P_{f}(V)=1-\exp \left[-\sum_{i=1}^{I P} \rho V_{i} p_{c, i}^{2}\left(\frac{r_{c, i}}{r_{0}}\right)^{1-\alpha}\right] .
$$

Note that $\rho_{i}=\rho p_{c, i}$ has been used in (16). Currently it contains three constant parameters that depend on the microstructure only: density of second-phase particles, $\rho$, minimum particle size, $r_{0}$, and shape of particle size distribution, $\alpha$.

The last step to construct the Weibull-stress based models is the selection of micro-crack propagation criterion, which determines the critical micro-crack size. The Beremin group [6] suggested two expressions for the critical micro-crack size. The first one is based on the Griffith criterion for crack stability in a linearly-elastic brittle material. For a penny shaped microcrack this criterion provides

$$
r_{c, i}=\frac{\pi E \gamma_{s}}{2\left(1-v^{2}\right) \sigma_{I, i}^{2}}
$$

where $\sigma_{I, i}$ is the maximum principal stress at the integration point $i$, and $\gamma_{s}$ is the surface energy density of the material. This criterion is suitable for failures that are not accompanied by significant plastic dissipation. This may include ferritic steels at very low temperatures, substantially below the ductile-to-brittle transition temperature. When a relatively large amount of the external work is dissipated in plasticity, Beremin suggested a correction to (17) that includes the plastic strain

$$
r_{c, i}=\frac{\pi E \gamma_{s} \exp \left(\varepsilon_{I, i}^{p}\right)}{2\left(1-v^{2}\right) \sigma_{I, i}^{2}}
$$

The correction was intended to account for the loss of crack driving force due to plastic dissipation. It essentially increases the critical micro-crack size and reduces the individual probability of failure as a result of increasing plasticity. Eqs. (17) or (18) are used in practically all Weibull-stress based local approaches. We will focus on (18), which after substitution in (15) gives the following individual failure probability at point $i$

$$
p_{f, i}=p_{c, i}\left(\frac{\sigma_{e f f, i}}{\sigma_{u}}\right)^{m},
$$

where $m=2 \alpha-2, \sigma_{e f f, i}$ is the effective stress at point $i$, and $\sigma_{u}$ is supposed to be a constant cleavage stress collecting all temperature independent terms in (19). The surface energy density, $\gamma_{s}$, may be assumed constant for the purposes of cleavage fracture modelling, because ab-initio calculations and experimental measurements for a number of metals and alloys demonstrate that it is independent on temperature within a large range between absolute zero and roughly half of the melting temperature, see e.g. [11]. The elastic modulus changes slightly with temperature and, if necessary, this change can be accommodated in the effective stress via the introduction of a reference modulus $E_{0}$, so that

$$
\begin{aligned}
& \sigma_{e f f, i}=\sigma_{I, i} \exp \left(-\frac{\varepsilon_{I, i}^{p}}{2}\right) \sqrt{\frac{E_{0}}{E}}, \quad \text { and } \\
& \sigma_{u}=\sqrt{\frac{\pi E_{0} \gamma_{s}}{2\left(1-v^{2}\right) r_{0}}} .
\end{aligned}
$$

The arrangement in (19)-(21) suggests that all temperature and loading dependence of the individual probability of failure is accommodated via the effective stress and the probability of micro-crack formation. The exponent $m$ and the cleavage stress $\sigma_{u}$ should remain constant. If the size distribution of microcrack initiating particles follows (14) with known (measured) parameters, (12) or (13) models appropriately the probability of micro-crack formation, and (18) models appropriately the plastic dissipation effect. Eq. (19) should then be able to predict accurately the individual probability of failure. Such predictions can be compared with experimentally measured locations of cleavage initiation sites. This will be illustrated in the next section.

With (19)-(21) the global (component) failure probability can be written in the following convenient form

$$
P_{f}(V)=1-\exp \left[-\sum_{i=1}^{I P} \frac{V_{i}}{V_{0}} p_{c, i}^{2}\left(\frac{\sigma_{e f f, i}}{\sigma_{u}}\right)^{m}\right],
$$

where $V_{0}$ is a volume scaling parameter. According to (16) it should be equal to $1 / \rho$, but in practice an arbitrary volume scale is used, while the density of the micro-crack initiating particles, being a constant, is incorporated into the cleavage stress, $\sigma_{u}$. Eq. (22) allows for writing the component probability of failure in terms of a Weibull distribution function of a global stress parameter, $\sigma_{w}$, with a scale $\sigma_{u}$ and a shape $m$ :

$$
\begin{aligned}
& P_{f}(V)=1-\exp \left[-\left(\frac{\sigma_{w}}{\sigma_{u}}\right)^{m}\right], \quad \text { with } \\
& \sigma_{w}=\sum_{i=1}^{I P} \frac{V_{i}}{V_{0}} p_{c, i}^{2} \sigma_{e f f, i}^{m} .
\end{aligned}
$$

If the temperature independent material parameters defining the scale $\sigma_{u}$ and the shape $m$ are known from experiment and the other model assumptions for particle failure and critical micro-crack size are sufficiently realistic, then (23) should be able to predict the experimentally observed toughness-temperature relation in the ductile-to-brittle transition region for high-constraint crack geometries. If the temperature 
independent parameters are not known, but the model assumptions are realistic, then a single calibration of the scale $\sigma_{u}$ and the shape $m$ should be sufficient for predicting the toughness-temperature dependence. Ideally, this could be done at any temperature and could be viewed as a process of determining the unknown temperature independent material parameters. Further, (23) with known parameters should be able to predict the cleavage fracture toughness of a material with the same micro-structural composition in terms of number density and size distribution of micro-crack initiating particles, i.e. the same scale $\sigma_{u}$ and shape $m$, in the cases when the mechanical fields in front of the macro-crack change. The reduction of crack-tip constraint in low-constraint geometry is certainly one such case. Irradiation-induced changes in the material yield and flow properties is also in this category, because there is no evidence to suggest that the radiation changes the density and size distribution of micro-crack initiating particles. Therefore, if (23) is sufficiently physically realistic, it is expected to predict irradiation- and constraintinduced cleavage fracture toughness changes at any temperature within the range of interest. The next section will show the predictions in terms of the reference temperature shifts and compare these with experimentally determined values.

\section{PREDICTIONS WITH WEIBULL STRESS MODELS}

The Weibull stress models do not rely on prior knowledge of the particle size distribution, but are rather based on calibrating the shape $m$ and the scale $\sigma_{u}$. It is well-known that for a high-constraint crack geometry the use of any $m$ within certain limits, e.g. $5<m<25$, will produce prediction for the probability of failure versus applied stress intensity at a fixed temperature in agreement with the Master Curve, provided that the scale parameter, $\sigma_{u}$, is calibrated for that temperature. We select two values of $m$ to illustrate the performance of the models, $m=22$ and $m=10$. In view of the models derivation, these correspond to two shapes of the particle size distribution, $\alpha=11$ and $\alpha=6$, respectively. For each $m, \sigma_{u}$ is determined as the Weibull stress, $\sigma_{w}$, attained at applied stress intensity equal to the reference toughness, $K_{0}$, at each temperature analysed. The predicted $\sigma_{u}$ dependence on temperature (unirradiated material) is shown in Fig. 1.

Clearly, none of the models is capable to produce a constant $\sigma_{u}$, but with the $\sigma_{u}$-temperature dependences shown all models are capable to predict the Master Curve toughnesstemperature dependence at any percentile with sufficient accuracy. Nevertheless, it is useful to compare the predicted toughness-temperature dependence with the Master Curve when a constant $\sigma_{u}$ calibrated at a single temperature is used across the whole temperature range. For illustration, we select $\langle\mathrm{M} 2\rangle$ and present the predictions with the two values of $m$ in Fig. 2 . The calibration of $\sigma_{u}$ is performed at $T_{0}$.
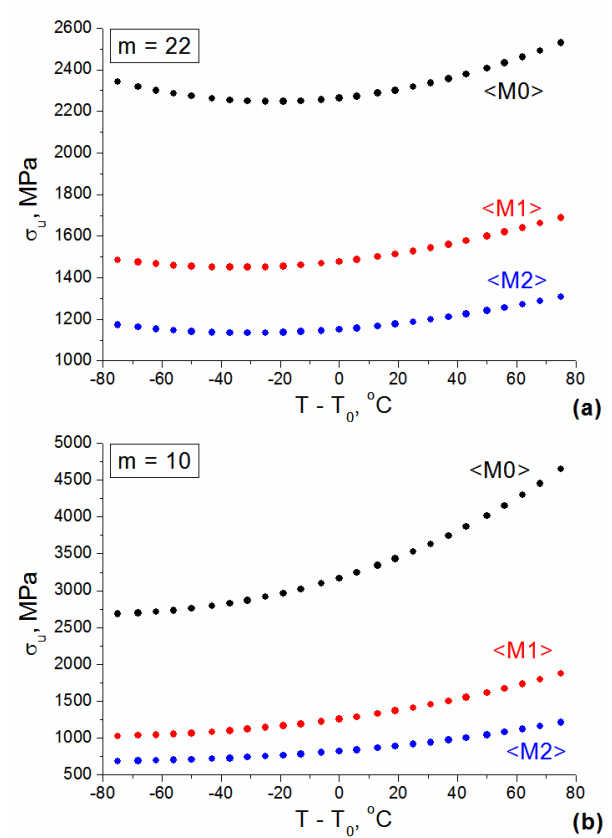

Figure 1. Temperature dependence of cleavage stress predicted by models: (a) with $m=22$; (b) with $m=10$.

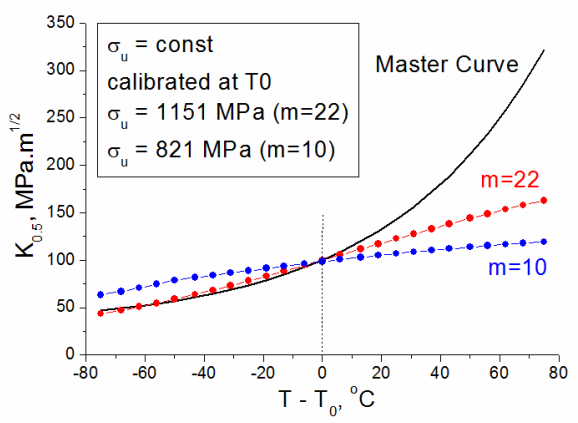

Figure 2. Temperature dependence of mean fracture toughness predicted by $\langle\mathrm{M} 2\rangle$ with constant $\sigma_{u}$.

Next, we test the models predictions for the individual probability of failure ahead of the macro-crack using (19). Results are presented in Fig. 3 for applied stress intensity equal to the reference toughness at the reference temperature only. Other cases are not significantly different from this. The results are compared with experimentally measured locations of cleavage initiation sites reported in [12] shown with green stars. The data points taken from [12] relate SEN(B) specimens with several crack lengths tested to fracture at several temperatures. To facilitate comparison the results are normalised so that the maximum individual failure probability is one. The results suggest that $\langle\mathrm{M} 2\rangle$ is superior for large $m$, but none of the models performs well for small $m$. In particular, $\langle\mathrm{M} 0\rangle$ is not capable to predict locations of cleavage initiation sites for any shape of particle size distribution. The outcome is no different if a constant $\sigma_{u}$ is used across the whole temperature range. 

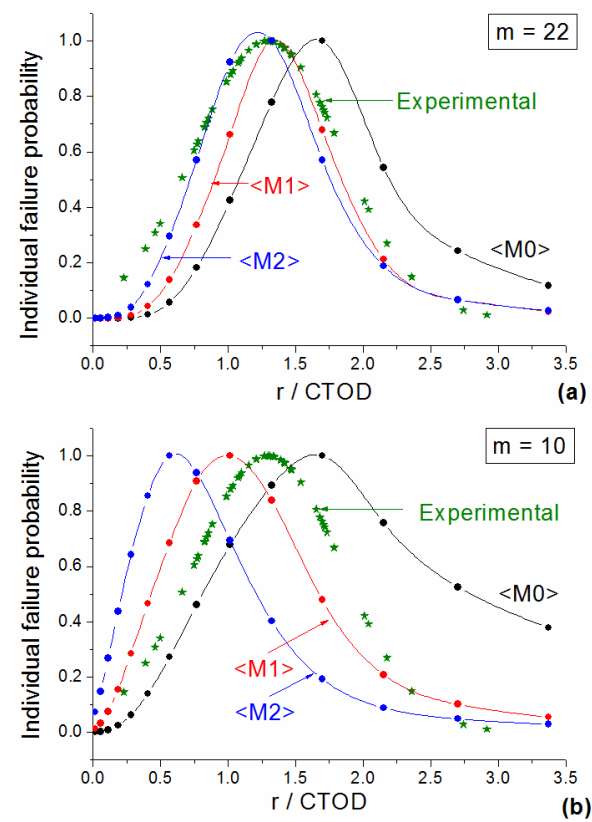

Figure 3. Individual failure probability predicted by models: (a) with $m=22$; (b) with $m=10$.

Finally, Fig. 4 shows the predictions of $\langle\mathrm{M} 2\rangle$ for the effects of irradiation and low-constraint on fracture toughness. In the figure, UH denotes unirradiated high-constraint prediction, UL denotes unirradiated low-constraint prediction, and IH denotes irradiated high-constraint predictions. For these predictions, the temperature dependent $\sigma_{u}$ is used, as shown in Fig. 1, hence UH prediction coincides with the Master Curve.
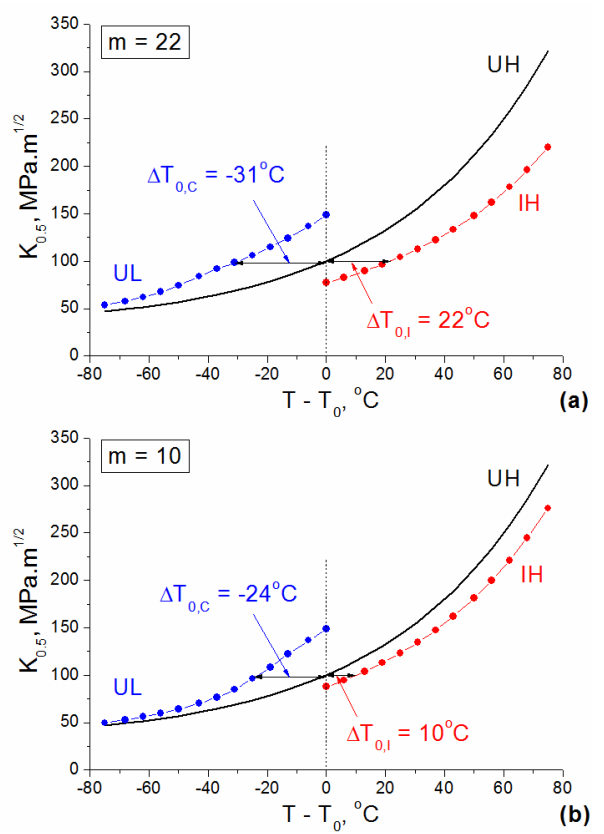

Figure 4. Predicted cleavage fracture toughness changes due to irradiation and constraint effects using $\langle$ M2 $\rangle$ : (a) with $m$ $=22$; (b) with $m=10$.
The predicted reference temperature shifts due to irradiation and low-constraint shown in Fig. 4 should be compared with the experimental values $\Delta T_{0, \mathrm{I}}=26^{\circ} \mathrm{C}$ and $\Delta T_{0, \mathrm{C}}=-24^{\circ} \mathrm{C}$, respectively. Clearly, the increase of $m$ yields an increase in the predicted detrimental effect of irradiation, but also an increase in the predicted beneficial effect of lowconstraint. A single $m$, however, cannot provide reasonably good predictions for the irradiation and the constraint effects simultaneously. Similar is the outcome when using the models $\langle\mathrm{M} 0\rangle$ and $\langle\mathrm{M} 1\rangle$. For the effect of irradiation, the three models provide nearly identical predictions with maximum of $1{ }^{\circ} \mathrm{C}$ difference with both $m$. For the effect of low-constraint, $\langle\mathrm{M} 1\rangle$ is nearly identical to $\langle\mathrm{M} 2\rangle$, while $\langle\mathrm{M} 0\rangle$ over-predicts the shift even more for $m=22$.

The formulation of the Weibull-stress based models makes the analysis of their behaviour difficult. In a sense these are global approaches that relay on a global parameter, the Weibull stress, to capture the complex collective behaviour of a set of micro-defects leading to cleavage failure. In the next section we propose a more micro-mechanically informed model that allows gaining further insight into the reasons for the deficiencies in the current local approach models.

\section{MICRO-MECHANICALLY INFORMED MODEL}

The model proposed below is based on an experimentally determined density and distribution of cleavage initiating particles in Material A [13]. Metallographic examination provided number density of particles $\rho=7.6 \times 10^{17} \mathrm{~m}^{-3}$. Further, it provided a probability density of particle sizes, which was fit to a Weibull-type statistics

$$
f(r)=\frac{\beta}{r_{0}}\left(\frac{r}{r_{0}}\right)^{-\beta-1} \exp \left[-\left(\frac{r}{r_{0}}\right)^{-\beta}\right],
$$

with $\beta=2.7$ being a shape parameter, and $r_{0}=0.036 \mu \mathrm{m}$ being a scale parameter representing the mean particle size.

The expression for the probability of particle failure is based on experimental observations that this probability depends not only on the mechanical fields but also on the particle size, similarly to the WST model [14]. The rationale is that larger particles have higher probability of failure than smaller particles under equal mechanical conditions. This goes a step further than the models (12) and (13) in describing the mechanism of micro-crack formation. The particles are assumed to be elastic-brittle with elastic constants equal to those of the matrix as a first approximation. The principle stresses, $\Sigma_{\alpha}$, in a particle can then be given in terms of the principal stresses, $\sigma_{\alpha}$, and plastic strains, $\varepsilon_{\alpha}^{p}$, in the matrix with

$$
\Sigma_{\alpha}=\sigma_{\alpha}+\frac{E}{1+v} \varepsilon_{\alpha}^{p}, \quad \alpha=1,2,3 .
$$


The criterion of particle failure is based on a critical value of the strain energy density in a particle associated with failure normal to the maximum principal stress. This is given by

$$
\psi_{c}=\frac{1+v}{E} \Sigma_{I}^{2}-\frac{v}{E} \Sigma_{I} \Sigma_{h}
$$

where $\Sigma_{I}$ and $\Sigma_{h}\left(=\sigma_{h}\right)$ are the maximum principal and the hydrostatic stress in the particle, respectively. If a particle of size $r$ ruptures normally to the maximum principal stress upon achieving some critical condition, the energy lost (or the work of rupture) will be proportional to $\Psi_{c}=r^{3} \cdot \psi_{c}$. The rupture of a particle is a stochastic event governed by the work of rupture and depending on the unknown particle constitution. The survival probability, $p_{s}$, of the particle must decrease with increasing work of rupture, which can be written in the form

$$
\frac{d p_{s}}{p_{s}}=-\frac{d \Psi_{c}}{\Psi_{0}}
$$

where $\Psi_{0}$ is a scaling energy. The survival probability at a given $\Psi_{c}$ can be determined by integrating (28) from the initial value of the work of rupture, which is zero, to the current value. The probability of particle rupture, $p_{c}$, is one minus the survival probability

$$
p_{c}=1-\exp \left(-\frac{\Psi_{c}}{\Psi_{0}}\right)=1-\exp \left[-\left(\frac{r}{r_{0}}\right)^{3} \frac{\psi_{c}}{\psi_{0}}\right]
$$

where the rupture energy density scale $\psi_{0}=\Psi_{0} / r_{0}{ }^{3}$ is introduced for convenience. Eq. (29) is used instead of (12) or (13) in the new model to determine the probability density of micro-cracks. Note, that (29) incorporates the effects of plastic strain and stress triaxiality. Increased plastic strains in the matrix will result in increased $\psi_{c}$ and hence probability of micro-crack formation under equal other conditions, see (26)(27). Inversely, increased hydrostatic stress, which could lead to micro-crack blunting, will result in reduced $\psi_{c}$ and hence probability of micro-crack formation under equal other conditions, see (26)-(27). Note also, that the particle size distribution (25) and the probability of micro-crack formation (29) do not allow for a closed form solution for the individual probability of failure (1). Numerical integration of (1) is therefore used for calculating the individual probabilities of failure at the integration points.

To accomplish the individual failure probability (1), a new expression for the critical micro-crack size is derived. The derivation is based on a heuristic argument that the behaviour of a micro-crack of radius $r$ formed in the plastic matrix corresponds to a fictitious micro-crack of radius $r_{f}>r$ in an elastic matrix. The crack opening displacement of a penny- shaped crack of radius $r_{f}$ in an elastic material subject to normal stress $\sigma_{I}$ is given by [15]

$$
u(x)=\frac{4\left(1-v^{2}\right) \sigma_{I}}{\pi E} \sqrt{r_{f}^{2}-x^{2}}, \quad 0<x<r_{f} .
$$

The blunting of the physical micro-crack formed after particle rupture can be approximated by $r \varepsilon^{p}$. The fictitious micro-crack size is then defined in such a way that its opening at $x=r$ equals the blunting of the physical micro-crack, i.e. $u(r)=r \varepsilon_{I}^{p}$. Solving (30) with this constraint leads to the following expression for the fictitious micro-crack size

$$
r_{f}=r \sqrt{1+\left[\frac{\pi E \varepsilon_{I}^{p}}{4\left(1-v^{2}\right) \sigma_{I}}\right]^{2}}
$$

This relation is used in conjunction with the Griffith criterion (17) to define an effective critical micro-crack size

$$
r_{c}=\frac{\pi E \gamma_{s}}{2\left(1-v^{2}\right) \sigma_{I}^{2}} \sqrt{1+\left[\frac{\pi E \varepsilon_{I}^{p}}{4\left(1-v^{2}\right) \sigma_{I}}\right]^{2}}
$$

Eq. (32) is used in the proposed model instead of (17) or (18) as a lower limit of the integral (1) which provides the individual probability of failure. The square-root factor in this proposal replaces the exponent of plastic strain in (18) suggested by the Beremin group. Similarly to [6], it is intended to account for the reduction in crack driving force due to plastic dissipation in the matrix. The difference is that in the case of (32) the effect is not only dependent on the plastic strain but also on stress triaxiality via the ratio between the maximum plastic strain and the maximum stress.

The model proposed in this section is implemented in a similar way to the Weibull-stress based models. However, because the individual probabilities of failure are calculated by numerical integration, an expression for the global probability of failure in terms of a global parameter, such as $\sigma_{u}$ in (23), is not possible. Therefore, the procedure calculates the global probability of failure based on (4). Results obtained with the proposed model are presented in the next section.

\section{PREDICTIONS WITH PROPOSED MODEL}

The aim with the proposed model is principally to achieve a reasonable description of the Master Curve with a single calibration at a given temperature. The shape $\beta$ and the average particle size $r_{0}$ are assumed to be known. One parameter that needs calibration is the rupture energy density scale $\psi_{0}$. This is calibrated so that the individual probability of failure profile ahead of the crack, calculated using (1) with (29) and (32), corresponds as closely to the experimental profile as possible. The calibrated value is $\psi_{0}=10^{5}$. With this value, Fig. 5 shows 
two profiles obtained with $\beta=2.7$ and $\beta=8$, corresponding to the experimentally determined shape and a larger shape used later in this section. For both profiles $r_{0}=0.036 \mu \mathrm{m}$ is used. As in Fig. 3 the profiles are compared to the experimental profile. Note, that unlike Fig. 3 the predicted profiles do not change significantly with the shape $\beta$.

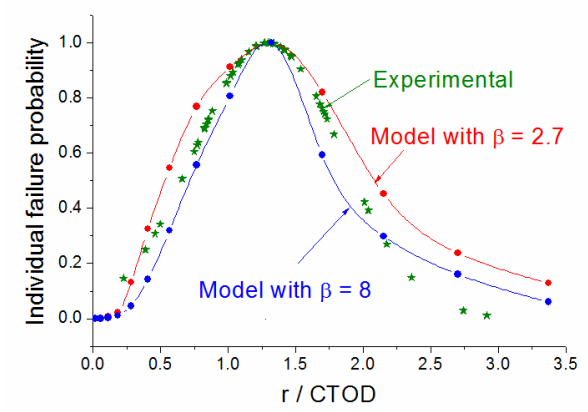

Figure 5. Individual failure probability predicted by proposed model with $\beta=2.7$ and $\beta=8$.

For calculating the global probability of failure via (4), a second parameter needs to be calibrated. This is a volume parameter, $V_{0}$, by which the elemental volumes are scaled, i.e. it scales the number of active micro-cracks to be accounted for in the calculation of the global probability of failure. In the proposed model this parameter is calibrated only once for a given $\beta$, at a selected temperature, and then used in the predictions across all temperatures studied. We have selected to perform this single calibration at the reference temperature. Using $\beta=2.7$ as suggested in [13], however, does not provide a satisfactory description of the Master Curve, as will be shown shortly.

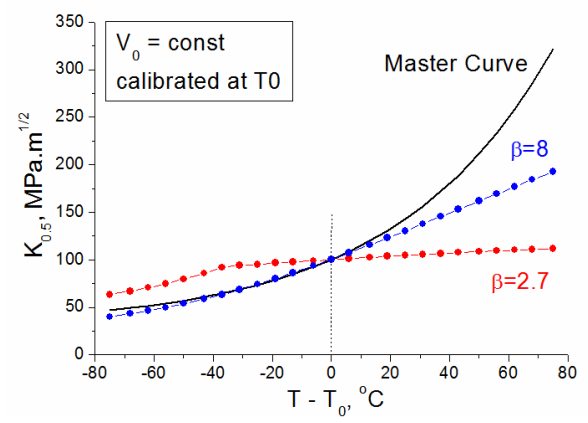

Figure 6. Temperature dependence of mean fracture toughness predicted by proposed model; calibration of volume scale performed at $T_{0}$ and used for all other temperatures.

By increasing $\beta$ it is possible to improve the prediction substantially. A reasonably good agreement with the Master Curve was found for $\beta=8$, which corresponds approximately to $m=14$ in the Weibull-stress models. The predicted toughnesstemperature dependences for this case as well as for $\beta=2.7$ are shown in Fig. 6. These predictions should be compared with the results in Fig. 2. Provided the proposed model is physically realistic, one possible reason for the very poor prediction with $\beta$ $=2.7$ is that this value may not be accurate, as being determined by curve fitting to a limited dataset. However, alternative explanation is the inability of the weakest-link statistics to account for all events leading to global failure.

The predictions of the proposed model for the cleavage fracture toughness changes with irradiation and constraint effects are shown in Fig. 7. The notations in the figure are identical to those in Fig. 4. However, the comparison now is made with the predicted toughness-temperature dependence for the unirradiated high-constraint case, UH. It should be noted, that all results are obtained with a single calibration of the volume scale, made for $\mathrm{UH}$ at reference temperature.

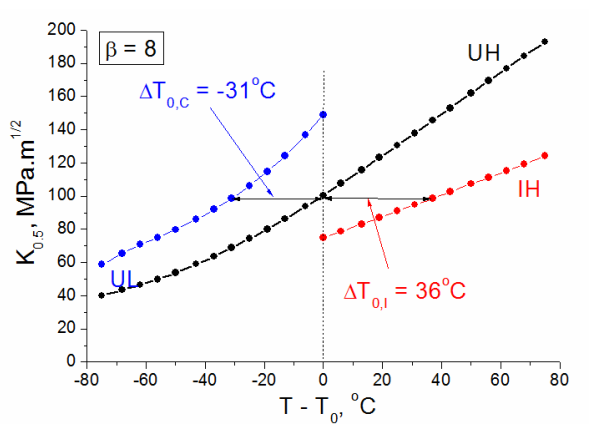

Figure 7. Predicted cleavage fracture toughness changes due to irradiation and constraint effects using the proposed model with $\beta=8$.

From these results it cannot be claimed that the proposed model is superior to the Weibull-stress based models when used with the large $m$, Fig. 4a. The proposed model notably overpredicts the detrimental effect of the irradiation; this however is on the safe side. It also over-predicts the beneficial effect of the low-constraint geometry to the same extent as the Weibull-stress based models. Nevertheless, the simulations performed with this model allowed for added insight into the effects of the various model components on the global probability of failure.

\section{DISCUSSION AND CONCLUSIONS}

The development of the local approach models for cleavage fracture has followed a path where the emphasis has been on improving the individual probability of failure as a function of the local mechanical fields. The way in which the global probability of failure, i.e. the failure probability of a cracked component, is calculated from such individual failure probabilities appears not to have been questioned during development of such models.

Using the weakest-link argument makes the formulation of the global failure probability admittedly more tractable, particularly when the individual probability of failure can be presented in closed form. Such is the case leading to the Weibull-stress based models assessed in this work. In these models, the individual failure probability is dictated by a powerlaw probability density of particle sizes and a probability of 
particle failure, i.e. micro-crack formation, which is independent of the particle size. The developments of the Weibull-stress models can be characterized in terms of improvements in estimation of the particle failure probability. The results presented in Fig. 3 suggest that these developments can yield improved predictions of the individual probability of failure ahead of the macro-crack when compared with experimentally measured locations of fracture initiation sites. This improvement, however, is strongly linked to the selected shape of the particle size distribution. The logic of the development of these models dictates that, for a given shape of the particle size distribution, the cleavage stress, which is the scale parameter of the models, should remain constant across all temperatures and for that matter for any other state for the same material. The change of the mechanical properties with temperature or with irradiation damage, as well as the change of the mechanical fields due to low-constraint geometry should be accounted for in the individual failure probabilities via the criteria for particle failure probability and critical micro-crack size. It is clear from Fig. 2 that the models assessed are not capable of predicting the Master Curve, i.e. the experimentally determined toughness-temperature dependence, with a constant scale for any reasonable shape of the particle size distribution.

In spite of this, the models can be used by calibrating the scale independently at each temperature, so that a cleavage stress vs. temperature dependence is established in advance, such as those shown in Fig. 1. By doing this, it is silently admitted that the individual probability of failure expressions used do not capture sufficiently well the dependence on the local mechanical fields. Monotonically increasing cleavage stress with temperature appears to be a logical way to account additionally for the increased plastic dissipation with increased temperature. It should be noted, however, that the dependence found for $m=22$ in Fig. 1a does not follow this logic. This suggests that the variation of the scale with temperature, needed to predict the experimentally determined toughness-temperature dependence, is not linked solely to increasing plasticity with temperature.

The use of the models with calibrated scale variation with temperature for predicting toughness changes due to irradiation and constraint effects reveals further deficiencies. Predicting the effect of irradiation with reasonable accuracy requires a large value of the shape parameter, Fig. 4a. This, however, over-predicts the effect of low-constraint. Predicting accurately the effect of a shorter crack on the other hand requires a smaller value of the shape parameter, Fig. 4b. This, in turn, leads to a substantial under-prediction of the detrimental effect of irradiation on cleavage fracture toughness. Reasonably good predictions for the two effects do not seem to be possible using a single shape parameter. Note, however, that according to the models' logical development, this parameter should remain constant for all cases of particle size distribution.

The new model, proposed in this work, offers an improvement of the criteria for the particle failure probability and for the critical micro-crack size. It is capable of predicting the individual probability of failure profile ahead of a macrocrack with good correspondence to experimental data, Fig. 5. The strength of the model is that it requires a calibration at a single temperature and the calibrated parameters can be used across all temperatures for predicting the Master Curve, Fig. 6, as well as for predicting irradiation and low-constraint effects, Fig. 7.

Because the new model requires a direct application of weakest-link statistics, it allows the analyst to uncover the effects of the various factors affecting the global probability of failure. The observations from such an analysis can be summarised as follows. At very low temperatures, substantially below the transition temperature, the number density of microcracks as predicted by the models for a particle failure probability at say 50\%-toughness is low. The corresponding individual events (micro-crack propagations with the corresponding individual failure probabilities) can be considered independent with good confidence, because of the relatively large distances between the micro-cracks. The weakest-link argument is a very good approximation to the failure statistics at these temperatures.

With an increasing temperature, the number density of the micro-cracks at the same toughness percentile increases and the distances between them decrease, due to increased plasticity and its effect on particle failure. The confidence in the independence of the corresponding individual events is reduced, because of the increased probability of micro-crack interaction. The interactions of the micro-cracks with increased plasticity progressively invalidate the use of weakest-link statistics for calculating the global failure probability. The interactions may have a two-way effect. On one hand, micro-cracks formed at some moment in the loading history but still stable at a later moment will affect the probability of failing non-ruptured particle in their vicinity. On the other, micro-cracks formed in the vicinity of a previously formed, and therefore longer, but still stable micro-crack will affect its probability of propagation. Thus, the collective behaviour of micro-cracks becomes increasingly important for the global outcome - the component probability of failure - as plasticity increases.

Further work is required to extend our knowledge of the collective behaviour of micro-cracks in a plastically deforming medium. This knowledge could be used for either reconsidering the weakest-link methodology for the global probability of failure or in developing a local approach for cleavage fracture based on an appropriate constitutive law for the behaviour of a material containing arrays of micro-cracks.

\section{REFERENCES}

[1] ABAQUS 6.9 User's Manual, Dassault Systems, 2009.

[2] Keim, E., 2005, "Summary of material data for 22NiMoCr37", Areva ANP GmbH, Technical Report.

[3] ASTM E 1921-05, "Standard Test Method for Determination of Reference Temperature $\mathrm{T}_{0}$ for Ferritic Steels in the Transition Range," ASTM 2005. 
[4] Williams, M.L., 1957, "On the stress distribution at the base of a stationary crack," J. Appl. Mech., 24, pp. 109-114.

[5] Sherry, A.H., France, C.C., Goldthorpe, M.R., 2005, "Compendium of T-stress solutions for two and three dimensional cracked geometries," Fatigue Fract. Eng. Mater. Struct., 18, pp. 141-155.

[6] Beremin, F.M., 1983, "A local criterion for cleavage fracture of a nuclear pressure vessel steel," Metal. Trans., A14, pp. 2277-2287.

[7] Xiaosheng, G., Zhang, G., Srivatsan, T.S., 2006, “A probabilities model for prediction of cleavage fracture in the ductile-to-brittle transition region and the effect of temperature on model parameters," Mater. Sci. Eng., A415, pp 264-272.

[8] Kroon, M., Faleskog, J., Oberg, H., 2008, “A probabilistic model for cleavage fracture with a length scale - Parameter estimation and predictions of growing crack experiments," Eng. Fract. Mech., 75, pp. 2398-2417.

[9] Hohe, J., Hardenacke, V., Luckow, S., Siegele, D., 2010, "An enhanced probabilistic model for cleavage fracture assessment accounting for local constraint effects," Eng. Fract. Mech., 77, pp. 3573-3591.

[10] Bordet, S.R., Karstensen, A.D., Knowles, D.M., Wiesner, C.S., 2005, "A new statistical local criterion for cleavage fracture of steel. Part I: model presentation," Eng. Fract. Mech., 72, pp. 435-452.

[11] Banerjea, A., Smith, J.R., 1988, "Origins of the universal binding-energy relation," Phys. Rev., B37, pp. 6632-6645.

[12] Hohe, J., Friedmann, V., Wenck, J., Siegele, D., 2008, "Assessment of the role of micro defect nucleation in probabilistic modeling of cleavage fracture," Eng. Fract.

Mech., 75, pp. 3306-3327.

[13] Ortner, S.R., Duff J., 2005, "Characterisation of Euro 'A' reference steel for application of $\mathrm{EOH}$ model of brittle fracture," Nexia Solutions Report.

[14] Wallin, K., Saario, T., Torronen, K., 1984, "Statistical model for carbide induced brittle fracture in steel," Metals Sci., 18, pp. 13-16. 